\title{
Correction of left ventricular asynchrony by coronary
} artery surgery

\author{
D G GIBSON, R A GREENBAUM, R B PRIDIE, M H YACOUB \\ From Harefield Hospital, Harefield, Middlesex, and Brompton Hospital, London
}

SUMMARY To investigate the effect of coronary artery bypass grafting on the timing of regional left ventricular wall motion, contrast left ventriculograms from 27 patients were digitised frame by frame before and after operation. End diastolic and end systolic volumes, ejection fraction, and peak ejection and filling rates showed no significant change. The commonest preoperative abnormality was delayed onset of inward wall motion during ejection, which was present in 14 patients over $10 \%$ (range 5-40\%) of the cavity outline, leading to a pattern of "diagonal contours". ㅇ After operation this pattern had resolved completely in 12 patients and partially in two. Minor $\rightarrow$ abnormalities appeared postoperatively in five but overall the mean (1SD) area affected was 3 reduced by $5(8) \%$. The time span between the onset of inward motion in different regions of the cavity also fell significantly after surgery from 190 (50) to 130 (50) ms. Regional hypokinesis (6 cases) and abnormal wall motion during isovolumic contraction (4 cases) or isovolumic relaxation ( 5 cases) were not consistently affected.

Thus successful coronary artery surgery is without consistent effect on overall left ventricular function, overall hypokinesis, or abnormal wall motion during the isovolumic periods. It does, however, strikingly reduce the asynchrony of wall motion during ejection, suggesting that before operation this abnormality may directly reflect impaired coronary blood flow. The results emphasise the potential value of analysing regional wall motion to elucidate functional abnormalities associated with coronary artery disease.

It is well established that coronary artery bypass grafting is associated with an increase in exercise tolerance in patients limited by angina of effort. Whether this improvement is accompanied by a corresponding change in resting left ventricular function is less clear. Although early results suggested an increase in ejection fraction, ${ }^{1}$ these findings have not been confirmed, and it is now generally accepted that basal measurements of overall function are not modified by operation in any clinically significant way. ${ }^{2}$ There is, however, little information about possible effects of surgery on the asynchronous wall motion so frequently present in patients with coronary artery disease.

It has been shown that wall motion abnormalities during pacing induced angina consistently arise in segments showing a characteristic pattern of asynchrony at rest. ${ }^{3}$ The possibility that this asynchrony at rest might be reversed by restoring normal coronary flow is clearly of potential clinical interest. The

Requests for reprints to Dr D G Gibson, Cardiac Department, Brompton Hospital, London SW3 6HP.

Accepted for publication 30 September 1987 present study was therefore undertaken to investigate the effects of bypass grafting on the timing of regional left ventricular wall motion in a group of patients in whom revascularisation had been successful.

\section{Patients and methods}

We studied 27 patients (mean (SD) age $52(7 \cdot 4)$ years; 25 men) who had had coronary artery bypass grafting with saphenous vein or internal mammary artery at Harefield Hospital. The patients were o selected only in so far as they had all undergone a $N$ preoperative and a routine postoperative study and $\underset{\omega}{\mathrm{N}}$ had right anterior oblique angiograms performed on these two occasions that were technically suitable for 0 analysis. Eight had had a myocardial infarction $\mathbb{D}$ before the first arteriogram-anterior in two and $\stackrel{?}{-}$ inferior in six. A mean (range) of 3.1 (1-5) grafts were 7 used, including an internal mammary artery to left $\stackrel{\vec{P}}{0}$ anterior descending coronary artery in 16 and to the right coronary artery in one. The interval between $\mathbb{\mathbb { D }}$ bypass grafting and the second catheterisation ranged between six and 695 days. All grafts were 
patent at restudy, and in no case was there any clinical, cardiographic, or enzyme evidence of intraoperative myocardial infarction. No patient had chest pain, ST segment change, or other evidence of acute myocardial ischaemia at the time of either study.

Angiograms were performed in the $30^{\circ}$ right anterior oblique position at a frame rate of $50 / \mathrm{s}$. Angiograms were calibrated with a rule exposed at mid-chest level. We did not study extrasystoles or postextrasystoles, and we calculated left ventricular volume using the area-length method for each cavity outline. ${ }^{4}$ Curves of ventricular volume versus time were smoothed with a three point moving average filter. We derived peak rates of left ventricular ejection and filling from this curve.

To quantify the extent and timing of regional wall motion 40 equally spaced points were identified by computer around the cavity outline with maximum area, and from each of these points the nearest point on the end systolic (minimum area) outline was identified. ${ }^{5}$ From each of the 40 lines thus identified, a plot of inward or outward endocardial motion against time was constructed. All 40 plots were combined to form a contour display (figure). Plots numbered 7-20 represent the inferior wall and 21-40 the free wall. Those from 1 to 6 represent the region of the mitral valve, and we disregarded them in assessing left ventricular wall motion. On each of the contour plots we identified the times of maximum and minimum cavity area and the onset of mitral valve opening, taken as the frame in which unopacified blood first appeared within the cavity. As in a previous study, ${ }^{6}$ we used an objective method to identify the onset of inward endocardial motion at the start of ejection. This was the beginning of a $100 \mathrm{~ms}$ period in which an inward velocity of at least $1 \mathrm{~cm} / \mathrm{s}$ was maintained throughout, starting during a time window stretching from $50 \mathrm{~ms}$ before the time of peak ventricular volume to mitral opening. This calculation was performed individually for each of the 40 segments around the cavity outline and the onset of motion was marked on the contour display. We noted the timing of peak inward wall motion for
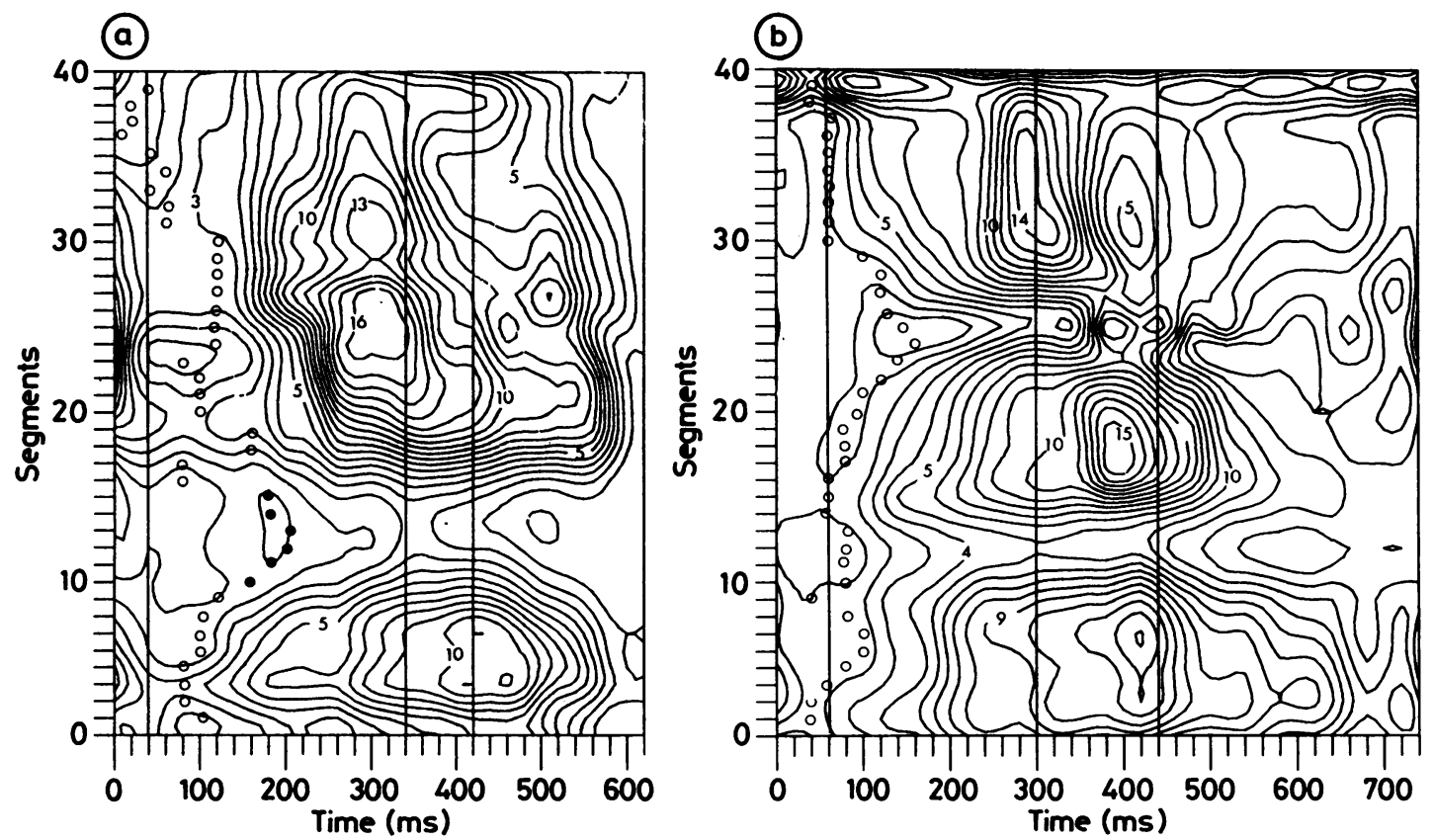

Fig (a) Contour display before operation from a patient with coronary artery disease. Contours represent endocardial position in $1 \mathrm{~mm}$ steps. Vertical lines represent maximum and minimum cavity volume and mitral valve opening. Circles represent the onset of inward motion for each segment, calculated as described in the text. Open circles indicate values within normal $95 \%$ confidence limits for the region; closed circles are values showing abnormal delay. The display indicates abnormal delay in the onset of inward motion in segments 10-15. The delay between the onset of inward motion in the earliest and latest segments around the cavity outline is increased to 200 ms. Segments 11-15 also show hypokinesis. Zero time is arbitrary. (b) Contour display from the same patient after operation. The timing of the onset of inward wall motion is now normal, and significant hypokinesis is no longer present. The time interval from earliest to latest onset of inward motion has dropped to $100 \mathrm{~ms}$. At the same time abnormal inward wall motion during isovolumic relaxation has now appeared in segments 17-23 associated with outward motion in segments 28-37. 
each segment in question, noting any values outside these limits.

We considered hypokinesis to be present when the overall amplitude of inward motion over three or more contiguous segments was less than the previously determined ${ }^{6}$ lower limit of normal for $95 \%$ confidence interval. We identified abnormalities of isovolumic contraction ${ }^{7}$ and isovolumic relaxation ${ }^{5}$ as previously described.

\section{STATISTICAL ANALYSIS.}

Differences in mean values were compared by Student's $t$ test either for groups or for paired values from the same patient before and after operation as appropriate. Values are expressed as mean (1 SD) throughout.

\section{Results}

OVERALL LEFT VENTRICULAR FUNCTION

The table gives the results in detail. Mean values for ventricular volumes, ejection fraction, and peak ejection and filling rates were all within normal limits before operation. In two patients, values for ejection fraction were reduced; one had had an old anterior infarction (ejection fraction $47 \%$ ) and the other an inferior infarction associated with cavity dilatation (ejection fraction $31 \%$ ). There was no significant change between preoperative and postoperative values for end diastolic and end systolic volumes, ejection fraction, or peak filling or ejection rates either when mean values were compared or when changes in individual patients were assessed. The minor increase in filling rate did not achieve statistical significance either when group means were compared or when values in individual patients were considered.

AMPLITUDE OF WALL MOTION

There were regions of hypokinesis in six patients before operation affecting 5-15 segments (13 to $38 \%$ of the cavity outline). After operation these had resolved in three and were unchanged in three.

TIMING OF THE ONSET OF INWARD WALL MOTION

In 14 patients (mean (range) segments $4(2-9)$ ) the

Table Overall left ventricular function (mean (1SD))

\begin{tabular}{lcc}
\hline & Preoperative & Postoperative \\
\hline End diastolic volume $(\mathrm{ml})$ & $120(37)$ & $110(38)$ \\
End systolic volume $(\mathrm{ml})$ & $40(24)$ & $36(21)$ \\
Ejection fraction $(\%)$ & $69(13)$ & $68(13)$ \\
Peak ejection rate $(\mathrm{ml} / \mathrm{s})$ & $420(150)$ & $450(220)$ \\
Peak filling rate $(\mathrm{ml} / \mathrm{s})$ & $440(140)$ & $540(260)$ \\
\hline
\end{tabular}

onset of inward wall motion was delayed with respect $\vec{c}$ to the timing of maximum volume. In eight patients $\vec{\Rightarrow}$ the mid-portion of the anterior wall was affected and $\stackrel{\oplus}{+}$ in six the distal portion of the inferior wall. Values for these segments when considered individually were $\frac{\bar{\sigma}}{\bar{C}}$ outside the normal $95 \%$ confidence interval, but they $\frac{\omega}{\sigma}$ were surrounded on either side by a region of relative $\varnothing$ delay, leading to a characteristic pattern of "diagonal $\risingdotseq$ contours" (figure). We estimated the overall extent of $\overrightarrow{0}$ this disturbance from the contour display, and found that it varied from six to 14 , mean (SD) 9.3 (3.1) $\vec{\omega}$ segments. After operation, the timing of the onset of $\frac{\mathrm{O}}{\partial}$ wall motion returned to within normal limits in 12 cases, and in the remaining two patients there was a 0 partial resolution (two segments each). In 13 patients $\omega$ the timing of the onset of inward wall motion with $\stackrel{\partial}{\perp}$ respect to maximum cavity volume was normal before operation. It remained so after operation in eight, but in five it became abnormal in 2-5 segments. $\vec{z}$ Overall, the mean number of abnormal segments per patient dropped by $1.5(2.8)$ after operation $(p<0.01$ 의 in paired $t$ test). This overall change was $2 \cdot 1(2 \cdot 4) \overrightarrow{0}$ when only patients with abnormal segments before or $\infty$ after operation were considered $(p<0.001)$. We also quantified the timing of the onset of inward motion by measuring the interval between its earliest and latest occurrence around the ventricle. Before operation this span was 190 (50) ms; it dropped to 130 (50) ms after operation. This fall was highly significant, $\varrho$ whether taken as the difference between the mean $\overrightarrow{\overrightarrow{0}}$ values $(p<0.01)$ or as individual changes $(p<0.001)$. 3

In five patients there was hypokinesis and delayed onset of motion in the same or overlapping segments. There was isolated delay in the onset of motion in $\vec{\sigma}$ nine and isolated hypokinesis in only one who had an old inferior myocardial infarction. In 12 patients we $\frac{\sigma}{5}$ found neither hypokinesis nor delay.

WALL MOTION DURING THE ISOVOLUMIC PERIODS

Outward wall motion was abnormal during $\triangle$ isovolumic contraction in four patients before operation. After operation it resolved in three. It appeared $\bar{N}$ de novo after operation in one. In two patients the inferior segments showed delayed inward motion 0 during isovolumic relaxation; in both this was $\tilde{\omega}$ relieved by operation. There was early outward wallo motion during isovolumic relaxation in threec patients; it was unaffected by surgery in all. It $\mathbb{D}$ appeared after operation as a new finding in three - $?$ along the anterior wall in one (figure) and the inferior $\frac{T}{T}$ wall in two. In one case abnormal outward motion $\frac{}{\mathbb{P}}$

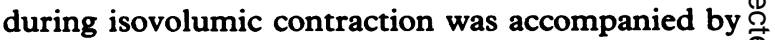
overall hypokinesis but, otherwise, abnormal motion $\propto$ during the two isovolumic periods was independent of hypokinesis or asynchrony during systole. Abnor- 8 
malities in the extent of wall motion during isovolumic relaxation did not alter the timing of peak inward wall motion, which was within normal limits for all patients before operation and remained so after surgery.

\section{Discussion}

\section{PREOPERATIVE FINDINGS}

Overall left ventricular function was well preserved in our patients: mean values for ventricular volumes, ejection fraction, and ejection and filling rates were all within normal limits. Even regional amplitude was preserved in all but six of the 27 patients, so that wall motion was normal on the basis of conventional angiographic analysis in most patients. When we used frame by frame analysis of angiograms to detect complex disturbances of timing, we identified abnormal function in more than half. This consisted of regional delay in the onset of inward motion, which we documented using objective criteria that took normal variation in timing into account. On the contour display the transition between normal regions and those showing delay in the onset of inward motion was not abrupt, but a recognisable pattern of diagonal contour lines was present, indicating gradual transition between the two. The whole disturbance affected nearly one quarter of the left ventricular cavity outline in the right anterior oblique projection. It was not consistently accompanied by any abnormality of wall motion during the two isovolumic periods, nor was the end of inward motion delayed. Thus even though overall left ventricular function was normal in these patients with angina pectoris caused by coronary artery disease, the timing of systolic wall motion was abnormal in most.

\section{POSTOPERATIVE FINDINGS}

The effects of successful coronary artery surgery were also consistent. Operation had no effect on ventricular volumes, ejection fraction, or peak rate of filling or ejection. It did, however, have a striking effect on the abnormal timing of wall motion. There was complete resolution in the delay in the onset of inward wall motion in 12 of 14 cases and partial resolution in the remaining two. Five new areas of abnormality appeared after operation, but these were less extensive, and overall there was a highly significant reduction in the number of abnormal segments. The improvement was even more clearly demonstrated when we quantified this asynchrony as the time interval between the earliest and latest onset of inward motion in different regions of the ventricle. After operation this interval fell by $30 \%$ to a value not significantly different from normal. Although there were disturbances of the two isovolumic periods of a type previously described in patients with chronic ischaemic heart disease, ${ }^{57}$ these were not consistently affected by operation. The present study thus confirms previous results in demonstrating no consistent effect of coronary artery bypass grafting on overall left ventricular function in patients who did not have evidence of acute ischaemia at the time of the first ventriculogram. ${ }^{28}$ Our observations are also compatible with previous echocardiographic studies on isovolumic wall motion that failed to demonstrate any improvement with antianginal drugs.' An effect of coronary bypass surgery in improving on the synchrony of ventricular wall motion during ejection has not received prominence in published reports, but the fact that it is a consistent finding when specifically sought suggests that it may be of pathophysiological importance.

In the present study we used contrast angiography because of its spatial resolution and frame rate. It does, however, have well documented disadvantages. We used only a single, right anterior oblique projection, so that we may have missed abnormalities in some cases. Although the overall extent of these disturbances of wall motion is considerable, the portion in which the onset of inward wall motion actually lies outside the normal limits is much smaller, so that when only the peripheral portion of the disturbance is demonstrated wall motion cannot be shown to be abnormal. It is also well recognised that the outer border of the ventricular cavity, as outlined by angiographic contrast medium, does not necessarily correspond to the position of endocardium. ${ }^{10}$ This discrepancy is greatest at end systole, however, whereas the crucial observations in the present study were made at end diastole and early systole when there had been little inward wall motion. The same applies to the possibilities of translation of the heart in space and rotation about its long axis. Perhaps the major problem in interpreting the results comes when one tries to define normality. In studies such as this it is usual to take results from patients in whom overall left ventricular function and coronary arteriograms are normal and in whom no other important intracardiac abnormality has been demonstrated. Yet such individuals usually present with angina, which in many cases is indistinguishable clinically from that associated with obstructive coronary artery disease. Their suitability as the standard of comparison for a study such as the present one that investigates more subtle abnormalities of wall motion, quite possibly closely related to the symptom of angina itself, can obviously be questioned. We have no answer to this objection, and accept that using a compromised standard will make any detection system less sensitive by widening the 
normal $95 \%$ confidence interval. Single plane contrast angiography thus seems to be an adequate method for demonstrating the disturbances we have described, although it may well underestimate their frequency.

The characteristic delay in the onset of inward motion at the onset of ejection in the absence of outward wall motion during isovolumic contraction has previously been described in patients with coronary artery disease. ${ }^{3}$ Its frequent occurrence under control conditions in regions that undergo akinesis during pacing induced angina and its predilection for the territory of the affected coronary artery in patients with single vessel coronary artery disease suggest that this behaviour is a marker of myocardium whose function is already compromised. The absence of outward wall motion during isovolumic contraction suggests that the timing of the onset of tension development was normal, with contraction initially isometric, indicating that the force generated was not great enough to cause inward motion. The absence of delay in the onset of outward wall movement demonstrates that the overall duration of contraction was normal. Its consistent improvement after bypass grafting confirms the close relation of abnormal wall motion to impaired blood flow and demonstrates its potential reversibility. Such behaviour can thus be regarded as a variant of "silent ischaemia", manifesting itself only by an abnormality of systolic wall motion without pain or even ST-segment change. Its response to revascularisation contrasts with the lack of change in segments moving abnormally during isovolumic contraction or relaxation, even when the two types of motion were present in the same ventricle.

A final question raised by these results is the nature of the link between abnormal wall motion and the symptom of angina. Angina occurs in conditions other than coronary artery disease. It is well recognised in hypertrophic cardiomyopathy and aortic stenosis, even when coronary arteriography shows the large vessels to be normal. It may thus be significant that in both these conditions similar studies of regional wall motion have demonstrated asynchronous onset of inward wall motion, unaccompanied by paradoxical outward motion during isovolumic relaxation or early ejection, and that in aortic stenosis this abnormality of wall motion is characteristically reversed by aortic valve replacement.. ${ }^{112}$ Angina and asynchronous wall motion are thus common factors in these two conditions, suggesting that the abnormal wall motion may be more than simply an independent marker of reduced coronary flow but may perhaps be more intimately related to the genesis of symptoms. Whatever the exact underlying mechanism, the present study gives some indication of the sensitivity $\stackrel{\stackrel{\mathrm{N}}{7}}{\mathrm{~T}}$ of regional wall motion in identifying abnormal $\stackrel{.}{-}$ patterns of wall motion seen in patients with coron- $\vec{s}$ ary artery disease, provided that the timing as well as $\overrightarrow{0}$ the amplitude of wall motion is taken into account. It suggests that such methods might be used to complement those based on regional metabolism or electrical abnormality in order to elucidate the complex pathogenesis of clinical disturbances associated ${ }^{\sigma}$ with myocardial ischaemia and coronary artery disease.

We thank Dr M K Towers for permission to report on patients admitted under his care.

\section{References}

1 Rees G, Bristow JD, Kremkau EK, et al. Influence of aortocoronary bypass surgery on left ventricular performance. N Engl J Med 1971;284:1116-20.

2 Hammermeister KE, Kennedy KW, Hamilton JE, et al. $\rightarrow$ Failure of successful grafting to improve resting left $\vec{z}$ ventricular function in chronic angina. $N \mathrm{Engl} \mathrm{J} \mathrm{Med}$ 1974;290:186-90.

3 Dawson JR, Gibson DG. Asynchronous left ventricular $\vec{\bullet}$ wall motion during pacing induced angina [Abstract]. $\infty$ Circulation 1986;74:(suppl II): 360.

4 Sandler $M$, Dodge HT. The use of single plane angiograms for the calculation of left ventricular volume in man. Am Heart $J$ 1968;75:325-40.

5 Gibson DG, Prewitt TA, Brown DJ. Analysis of left ventricular wall movement during isovolumic relaxation and its relation to coronary artery disease. $\mathrm{Br}$ Heart $J$ 1976;38:1010-9.

6 Hammermeister KE, Gibson DG, Hughes D. Regional variation in the timing and extent of left ventricular wall motion in normal subjects. $\mathrm{Br}$ Heart $J$ 1986;56:226-35.

7 Gibson DG, Doran JH, Traill TA, Brown DJ. Regional abnormalities in left ventricular wall movement during isovolumic relaxation in patients with ischaemic 3 heart disease. Eur J Cardiol 1978;7(suppl):251-64.

8 Chatterjee K, Swan HJC, Parmley WW, Susaita H, 응 Marcus H, Matloff J. Depression of left ventricular

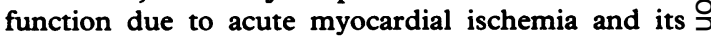
reversal after aortocoronary saphenous-vein bypass. N Engl J Med 1972;286:1117-21.

9 Hall RJC, Doran J, Pusey C, McHaffie D, Gibson DG. The effect of nitroglycerin, beta blockade with ? acebutalol and isometric stress on incoordinate left $\tilde{O}$ ventricular function. Eur Heart $J$ 1982;3:23-8.

10 Hugenholtz PG, Kaplan K, Hull E. Detection of left ventricular wall thickness by angiocardiography. $\mathrm{Am}$ Heart J 1969;78:513-22.

11 Hammermeister KE, Gibson DG, Hughes D, Jones $\mathbb{D}$ RH. Coordination of left ventricular wall motion ? before and after valve replacement for aortic stenosis [Abstract]. Circulation 1985;72: (suppl III): 367.

12 Gibson DG, Sanderson JE, Traill TA, Brown DJ, $\stackrel{\square}{\Omega}$ Goodwin JF. Regional left ventricular wall movement $\mathbb{D}$ in hypertrophic cardiomyopathy. $\mathrm{Br}$ Heart $J \frac{\mathrm{Q}}{\mathrm{O}}$ 1978;40:1327-33. 